\title{
Avaliação da resistência da soja a Sclerotinia sclerotiorum em diferentes estádios fenológicos e períodos de exposição ao inóculo
}

\author{
Riccely A. Garcia ${ }^{1}$ \& Fernando C. Juliatti \\ 'Departamento de Fitossanidade, Universidade Federal de Goiás, Cx. Postal 131, 74001-970, Goiânia, GO, Brasil; ${ }^{2}$ Laboratório \\ de Micologia e Proteção de Plantas (LAMIP), Instituto de Ciências Agrárias, Universidade Federal de Uberlândia, Campus \\ Umuarama, sala 106, bloco 2 E, 38400-902, Uberlândia, MG, Brasil
}

Autor para correspondência: Fernando C. Juliatti, e-mail: juliatti@ufu.br

\begin{abstract}
RESUMO
Os objetivos deste trabalho foram determinar o estádio fenológico ideal de plantas de soja para inoculação de Sclerotinia sclerotiorum, bem como o período apropriado de contato do inóculo com o tecido da planta para detectar resistência parcial de cultivares de soja ao patógeno. Foram testadas duas cultivares de soja, MG/BR-46 (Conquista) e M-SOY 8200, com plantas em cinco estádios fenológicos diferentes $\left(\mathrm{V}_{1}, \mathrm{~V}_{2}, \mathrm{~V}_{3}, \mathrm{~V}_{4}\right.$ e $\left.\mathrm{R}_{1}\right)$ e a inoculação envolveu diferentes períodos de contato de discos de cultura de um isolado de S. sclerotiorum escolhido por sua elevada agressividade à soja. Observou-se que a severidade da doença decresceu em proporção direta à idade das plantas, determinando-se $V_{1}$ e $V_{2}$ (folhas e hastes) e $V_{3}$ (folhas) como estádios ideais de inoculação para a avaliação de resistência de cultivares de soja. O contato contínuo do inóculo com o tecido vegetal proporcionou bons resultados para a inoculação de S. sclerotiorum, tanto em órgãos destacados como em órgãos ligados às plantas. Foram avaliados 90 genótipos de soja em inoculações sobre folhas destacadas pela colocação de discos de cultura em contato permanente com os folíolos das plantas. Apenas 19 genótipos comportaram-se como resistentes ou moderadamente resistentes e, dentre estes, apenas dois foram moderadamente resistentes em inoculações nas plantas.
\end{abstract}

Palavras-chave: Glycine max, estádios fenológicos, podridão branca da haste, resistência.

\begin{abstract}
Evaluation of soybean resistance to Sclerotinia sclerotiorum at different phenological stages and over different periods of exposure to inoculum

The objectives of this work were to determine the most adequate phenological stage of soybean plants for inoculation of Sclerotinia sclerotiorum and the period of contact between inoculum and plant tissues to detect partial resistance of soybean to the pathogen. Plants of two soybean cultivars, MG/BR-46 (Conquista) and M-SOY 8200 were inoculated at five different phenological stages $\left(\mathrm{V}_{1}, \mathrm{~V}_{2}, \mathrm{~V}_{3}, \mathrm{~V}_{4}\right.$ and $\mathrm{R}_{1}$ ). Inoculation involved different periods of contact between culture disks of an isolate of $S$. sclerotiorum which was selected based on high aggressiveness against soybean. It was observed that disease severity decreased as plants became older and it was concluded that $\mathrm{V}_{1}$ and $\mathrm{V}_{2}$ (leaves and stems) and $\mathrm{V}_{3}$ (leaves) were ideal stages for inoculation during tests aimed at determining levels of resistance in soybean cultivars. Continuous contact of inoculum with plant tissues yielded good results for inoculation of $S$. sclerotiorum, either onto detached organs or onto organs connected to living plants. Ninety genotypes of soybean were evaluated by placing culture disks in continuous contact with folioles. Only 19 genotypes were moderately resistant and, among these, only two were moderately resistant in inoculation involving whole plants.
\end{abstract}

Key words: Glycine max, phenological stages, resistance, Sclerotinia stem rot.

\section{INTRODUÇÃO}

A podridão branca da haste, causada pelo fungo Sclerotinia sclerotiorum (Lib.) de Bary, tem se tornado uma doença de grande importância para a cultura da soja em muitas regiões do Centro-Sul do Brasil. Isto se deve principalmente à alta precipitação pluvial durante a safra, aliada a temperaturas amenas, rotação de culturas com espécies altamente suscetíveis (Leite, 2005) e ao uso de sementes contaminadas, produzidas pelos próprios agricultores (Juliatti \& Juliatti, 2010). A fase mais vulnerável da planta compreende os estádios da floração plena $\left(\mathrm{R}_{2}\right)$ ao início da formação das vagens $\left(\mathrm{R}_{3} / \mathrm{R}_{4}\right)$ (Almeida et al., 2005). Os sintomas ocorrem geralmente no terço médio das plantas, atingindo haste principal, pecíolos, folhas e vagens.

Diferenças entre cultivares de soja quanto à reação a $S$. sclerotiorum têm sido avaliadas no campo, casa de vegetação e laboratório, sendo observadas respostas que variam de elevada resistência a completa suscetibilidade (Homechin, 1983; Boland \& Hall, 1987; Silva \& Machado, 1989; Wegulo et al., 1998; Chaves et al., 1996). Para seleção 
de genótipos de soja quanto à resistência a S. sclerotiorum é necessária a utilização de métodos de inoculação confiáveis, bem como a identificação de qual estádio fenológico é mais adequado para inoculação. Vários métodos de inoculação em condições ambientais controladas têm sido avaliados. Chaves et al. (1996) avaliaram a inoculação de $S$. sclerotiorum em diferentes densidades e locais de inoculação (junto à semente, junto ao colo de plantas jovens e no solo), por meio do uso de micélio seco e escleródios em plantas de soja. Os resultados revelaram que micélio seco, quando depositado na superfície do solo, junto ao colo de plantas jovens, foi o mais eficiente para seleção de genótipos de soja. Com a técnica de inoculação usando micélio seco, os autores verificaram que a cultivar FT-Abyara mostrou maior resistência e a cultivar Cobb maior suscetibilidade, enquanto que BR-4, BR-16 e IAS-5 situaram-se em uma faixa intermediária. Apesar da variabilidade genética da soja para resistência a $S$. sclerotiorum já ter sido relatada na literatura, pouco se sabe sobre a variabilidade no germoplasma brasileiro. Também há pouca informação sobre métodos de inoculação adequados para seleção de genótipos de soja resistentes à doença. Este trabalho teve como objetivo determinar o estádio fenológico ideal de plantas de soja para inoculação de $S$. sclerotiorum, bem como o período apropriado de contato do inóculo com o tecido da planta para detectar resistência parcial de cultivares de soja ao patógeno.

\section{MATERIAL E MÉTODOS}

Foram estudados os estádios $\mathrm{V}_{1}, \mathrm{~V}_{2}, \mathrm{~V}_{3}, \mathrm{~V}_{4}$ e $\mathrm{R}_{1}$ das cultivares BR/MG-46 (Conquista) e M-SOY 8200, em delineamento inteiramente casualizado em esquema fatorial de 5 (estádios) x 2 (órgãos) x 2 (cultivares), com três repetições. Um isolado de Jataí, GO foi previamente selecionado pela maior agressividade em plantas inoculadas. As inoculações foram realizadas em folhas e hastes. No estádio $\mathrm{V}_{1}$ foram inoculadas as folhas opostas; $\mathrm{em} \mathrm{V}_{2} \mathrm{e} \mathrm{V}_{3}$, o $1^{\circ}$ trifólio; em $\mathrm{V}_{4}$, o $2^{\circ}$ trifólio; e em $\mathrm{R}_{1}$, o $3^{\circ}$ trifólio. As inoculações nas hastes foram realizadas no $2^{\circ}$ internódio a partir da gema apical e em somente um dos folíolos (face abaxial). Os folíolos e as hastes foram inoculados com discos de culturas formadas em batata-dextrose-ágar (BDA) com $6 \mathrm{~mm}$ de diâmetro contendo micélio cultivado por sete dias a temperatura de $22 \pm 3^{\circ} \mathrm{C}$. Os discos foram depositados com o micélio em contato direto com o folíolo e a haste e fixados com fita adesiva. Posteriormente, as plantas foram mantidas em câmara de crescimento a $22 \pm 3^{\circ} \mathrm{C}$ e 12 horas de fotoperíodo com luz fluorescente, durante 72 horas.

Dois experimentos foram conduzidos para avaliar os métodos de inoculação, um em hastes e folhas destacadas e outro em plantas inteiras das cultivares MG/BR-46 (Conquista) e M-SOY 8200. Com discos de cultura de $S$. sclerotiorum, os métodos testados foram: "discopermanente" (o disco permaneceu nos órgãos inoculados até o momento da avaliação de severidade), "disco 24 horas" (o disco foi retirado do centro dos folíolos e dos internódios 24 horas após a inoculação) e "disco toque" (o disco foi depositado e retirado em seguida), em delineamento inteiramente casualizado em fatorial de 3 (métodos) x 2 (órgãos) x 2 (cultivares), com três repetições. As inoculações foram em plantas no estádio $\mathrm{V}_{2}$, cultivadas em copos plásticos com capacidade para $500 \mathrm{~mL}$, contendo substrato orgânico para produção de mudas de tomateiro (Plantcell), os quais foram mantidos em casa de vegetação. Para os órgãos destacados, os três folíolos correspondentes ao $2^{\circ}$ trifólio, de cada planta de cada genótipo ou cultivar, e o $2^{\circ}$ internódio foram colocados separadamente em caixas gerbox, contendo quatro folhas de papel toalha umedecidas em água destilada estéril, em ambiente asséptico. As inoculações foram feitas como descrito anteriormente. Após a inoculação, as caixas contendo os folíolos e as hastes destacadas, bem como as plantas inteiras foram mantidas em câmara de crescimento, nas mesmas condições descritas anteriormente.

O experimento para testar a reação de genótipos de soja a $S$. sclerotiorum foi conduzido em delineamento inteiramente casualizado, com três repetições. De 90 cultivares previamente classificadas como resistentes e moderadamente resistentes, em inoculações em folha destacada (Tabela 4), as seguintes foram selecionadas para inoculação em folhas de plantas inteiras: Emgopa 316, BRSGO Milena, Emgopa 314, FMT Perdiz, FMT Tabarana (cultivar mais suscetível pelo teste com folha destacada), M-SOY 8360, BR 16, BRSMG 790A, P98R31, M-SOY 8352, M-SOY 2002, BRSMG 68 (Vencedora), BRSGO Princesa, CD 211, CD 205, BRSGO Caiapônia, M-SOY 8008, BRS 185, Emgopa 315 e BRS Baliza RR. Estas cultivares, ao atingirem o estádio $\mathrm{V}_{3}$, foram inoculadas como previamente descrito pela técnica do "disco permanente" em um dos folíolos do $1^{\circ}$ trifólio.

Em todos os experimentos, as avaliações da severidade nas folhas inoculadas foram realizadas 72 horas após a inoculação, com base em escala diagramática (Garcia \& Juliatti, 2008), com a utilização do Programa Quant (Vale et al., 2003) (Figura 1). Foram utilizados 400 folíolos com níveis diferentes de porcentagem de área necrosada em decorrência da infecção por S. sclerotiorum, variando de 0 a $100 \%$, para estimar as severidades e definir os intervalos de avaliação pela escala diagramática. As cultivares foram classificadas como imunes (ausência de doença), resistentes (severidade de 0 a 12\%), moderadamente resistentes (severidade de 12 a 25\%), moderadamente suscetíveis (severidade de 25 a 50\%) e suscetíveis (severidade maior que $50 \%$ ). Para avaliação da severidade na haste calculouse a porcentagem de doença em relação ao comprimento total da haste, em que: $\%$ doença $=$ comprimento da lesão $(\mathrm{cm}) /$ comprimento total da haste $(\mathrm{cm}) \times 100$. Os dados de severidade da doença (área foliar doente) foram submetidos à análise de variância e as médias comparadas pelo teste de Tukey, para os experimentos de estádios fenológicos e métodos de inoculação, e teste de Scott-Knott, para o experimento de reação de cultivares, utilizando-se o 


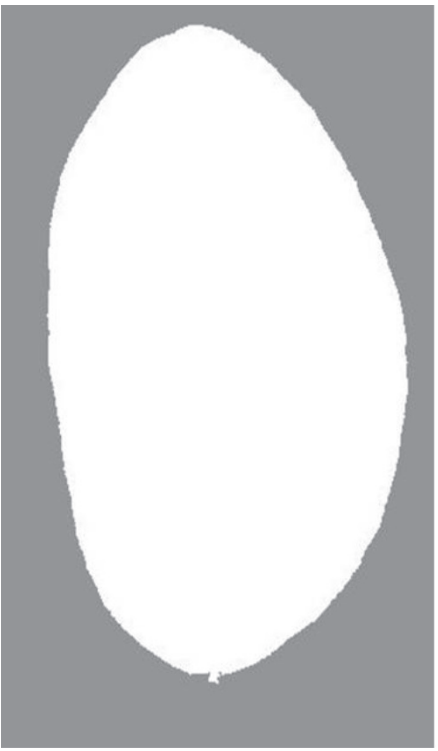

$0 \%$

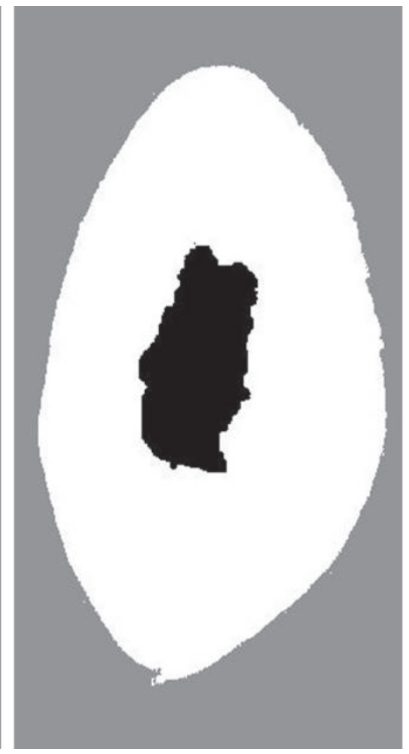

$12 \%$

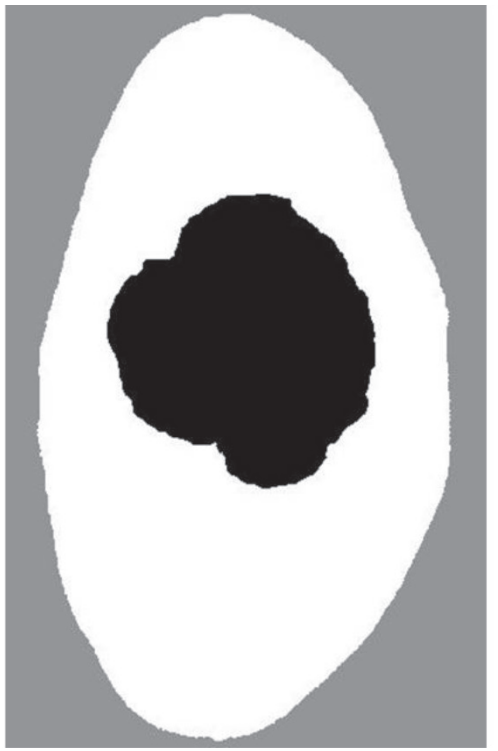

$25 \%$

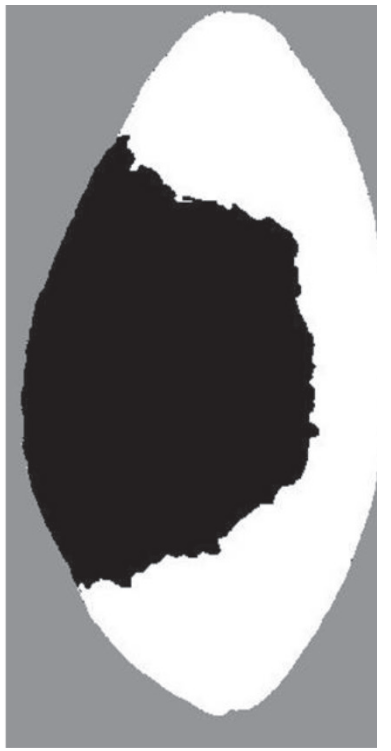

$50 \%$

FIGURA 1 - Escala diagramática para avaliação de sintomas de Sclerotinia sclerotiorum em folíolos de soja (Glycine max) (Garcia \& Juliatti, 2008)

software Sisvar. As análises de correlação para métodos de inoculação entre órgãos destacados e na planta e de reação das cultivares inoculadas em folhas destacadas e na planta foram realizadas pelo teste $t$.

\section{RESULTADOS E DISCUSSÃO}

As porcentagens médias de severidade da doença nas cultivares MG/BR-46 (Conquista) e M-SOY 8200 decresceram com o avanço do estádio fenológico (Tabela 1), concordando com os resultados obtidos por Chun et al. (1987). Chaves (1995) também constatou um acréscimo linear na taxa de sobrevivência, à medida que as plantas avançaram em seu desenvolvimento. Em razão de os valores de severidade terem sido mais próximos entre inoculações nos folíolos e nas hastes para ambas as cultivares, os estádios $\mathrm{V}_{1}$ ou $\mathrm{V}_{2}$ possivelmente são os mais indicados para inoculação de S. sclerotiorum em plantas de soja, para fins de avaliação de resistência à podridão branca da haste. Considerando inoculações somente em folíolos, o estádio $\mathrm{V}_{3}$ apresentou resultados similares ao estádio $\mathrm{V}_{2}$.

Independente das inoculações nos órgãos destacados ou na planta, os métodos de "disco permanente" e "disco 24 horas" resultaram em maior severidade da doença para ambas as cultivares e para os órgãos (Tabelas 2 e 3). A diferença entre as cultivares, quanto à suscetibilidade à doença, foi observada somente entre os métodos de "disco toque" e "disco 24 horas" na haste. A maior severidade da doença foi observada quando o órgão inoculado foi o folíolo, independente da cultivar utilizada. A exceção ocorreu somente no método de "disco toque", para a cultivar MG/BR-46 (Conquista), e "disco 24 horas", para a cultivar MSOY 8200, em que a inoculação na haste resultou em maior severidade. $\mathrm{O}$ fato de os folíolos terem sido mais suscetíveis pode ser explicado pelo menor acúmulo de lignina em relação às hastes. Entre as desvantagens do método "disco 24 horas" mencionam-se a mão de obra necessária para retirá-los e os ferimentos ocasionados nas folhas em função da fita adesiva usada para fixá-los. O método do "disco permanente" apresentou mais praticidade em relação aos demais métodos. Além disso, esse método aproxima-se mais da condição de campo, pois quando o inóculo alcança a superfície da planta, aí permanece por tempo indeterminado. A comparação entre as inoculações nos órgãos destacados e na planta proporcionou uma correlação $(r=0,9189)$ altamente significativa $(p<0,01)$. Isto comprova que o método de inoculação em órgãos destacados é adequado para seleção de genótipos de soja à S. sclerotiorum. Com este método, pode-se estudar a reação de vários genótipos para seleção inicial, com economia de tempo e espaço, comparando-se com o método de inoculação na planta.

As cultivares apresentaram comportamento variado quanto à reação a S. sclerotiorum. Das 90 cultivares avaliadas em inoculações nas folhas destacadas, dez comportaram-se como resistentes, sete como moderadamente resistentes, sete como moderadamente suscetíveis e 66 como suscetíveis. Entre as cultivares classificadas como resistentes, Emgopa 316, BR 16 e BRSGO Milena foram as que apresentaram menor severidade da doença (Tabela 4). As cultivares que foram resistentes e moderadamente resistentes pelo método da folha destacada não se comportaram da mesma forma 
Avaliação da resistência da soja a Sclerotinia sclerotiorum em diferentes estádios...

TABELA 1 - Porcentagem de área necrosada decorrente da infecção por Sclerotinia sclerotiorum em função de órgãos inoculados e estádios fenológicos de plantas de soja. Médias de seis repetições, com as cultivares MG/BR46 (Conquista) e M-SOY 8200

\begin{tabular}{lccccc}
\hline \hline Órgão & \multicolumn{5}{c}{ Estádios } \\
\cline { 2 - 6 } & $\mathbf{V}_{\mathbf{1}}$ & $\mathbf{V}_{\mathbf{2}}$ & $\mathbf{V}_{\mathbf{3}}$ & $\mathbf{V}_{\mathbf{4}}$ & $\mathbf{R}_{\mathbf{1}}$ \\
\hline Folha & $100 \mathrm{a} \mathrm{A}$ & $100 \mathrm{a} \mathrm{A}$ & $98,8 \mathrm{a} \mathrm{AB}$ & 77,5 a B & 41,2 a C \\
Haste & $95,3 \mathrm{a} \mathrm{A}$ & $62,3 \mathrm{~b} \mathrm{~B}$ & $25,3 \mathrm{~b} \mathrm{C}$ & $29,2 \mathrm{~b} \mathrm{C}$ & $18,2 \mathrm{~b} \mathrm{C}$ \\
\hline CV $(\%)$ & & & 20,87 & \\
\hline
\end{tabular}

Médias seguidas de letras distintas minúsculas nas colunas e maiúsculas nas linhas diferem entre si, pelo teste de Tukey, a 1\% de probabilidade.

TABELA 2 - Porcentagem de área necrosada decorrente da infecção por Sclerotinia sclerotiorum em função do método de inoculação em órgãos destacados de soja

\begin{tabular}{|c|c|c|c|c|}
\hline \multirow[t]{2}{*}{ Método } & \multicolumn{2}{|c|}{ MGBR46 (Conquista) } & \multicolumn{2}{|c|}{ M-SOY 8200} \\
\hline & Folíolo & Haste & Folíolo & Haste \\
\hline Testemunha & 0,0 a A & 0,0 a $\mathrm{A}$ & 0,0 a $\mathrm{A}$ & 0,0 a $\mathrm{A}$ \\
\hline "Disco toque" & 4,3 a $\mathrm{A}$ & $25,0 \mathrm{~b}$ B & 2,3 a A & 0,0 a $\mathrm{A}$ \\
\hline "Disco 24 horas" & $77,3 \mathrm{~b}$ B & $44,0 \mathrm{c} \mathrm{A}$ & 85,7 b A & 100,0 c B \\
\hline "Disco permanente" & 91,3 c A & $84,0 \mathrm{~d} A$ & 91,7 b B & $80,3 \mathrm{~b} \mathrm{~A}$ \\
\hline
\end{tabular}

Médias seguidas de letras distintas minúsculas nas colunas mostram diferenças significativas para métodos dentro de variedades e órgãos e letras maiúsculas nas linhas mostram diferenças significativas para órgãos dentro de variedades e métodos, pelo teste de Tukey a 5\% de probabilidade. "Disco toque" - disco de cultura depositado sobre órgão da planta e retirado em seguida; "Disco permanente" - disco de cultura depositado e mantido continuamente sobre órgãos inoculados até o momento da avaliação de severidade; "Disco 24 horas" - disco de cultura depositado sobre o centro de folíolos ou sobre hastes e ali mantidos por 24 horas e então removidos.

TABELA 3 - Severidade da infecção de Sclerotinia sclerotiorum (\% de área necrosada) em função do método de inoculação em plantas de soja

\begin{tabular}{|c|c|c|c|c|}
\hline \multirow[t]{2}{*}{ Método } & \multicolumn{2}{|c|}{ MG/BR46 (Conquista) } & \multicolumn{2}{|c|}{ M-SOY 8200} \\
\hline & Folíolo & Haste & Folíolo & Haste \\
\hline Testemunha & 0,0 a $\mathrm{A}$ & 0,0 a $\mathrm{A}$ & 0,0 a $\mathrm{A}$ & 0,0 a A \\
\hline "Disco toque" & 0,0 a $\mathrm{A}$ & $0,0 \mathrm{~b} \mathrm{~A}$ & 0,0 a $\mathrm{A}$ & 0,0 a A \\
\hline "Disco 24 horas" & $94,0 \mathrm{~b} \mathrm{~B}$ & $48,0 \mathrm{~b} \mathrm{~A}$ & 89,7 b B & $58,0 \mathrm{~b} A$ \\
\hline "Disco permanente" & $96,0 \mathrm{~b} \mathrm{~B}$ & $50,7 \mathrm{~b} \mathrm{~A}$ & 98,7 c B & $63,0 \mathrm{~b} \mathrm{~A}$ \\
\hline
\end{tabular}

Médias seguidas de letras distintas minúsculas nas colunas mostram diferenças significativas para métodos dentro de variedades e órgãos e letras maiúsculas nas linhas mostram diferenças significativas para órgãos dentro de variedades e métodos, pelo teste de Tukey a $5 \%$ de probabilidade. "Disco toque" - disco de cultura depositado sobre órgão da planta e retirado em seguida; "Disco permanente" - disco de cultura depositado e mantido continuamente sobre órgãos inoculados até o momento da avaliação de severidade; "Disco 24 horas" - disco de cultura depositado sobre o centro de folíolos ou sobre hastes e ali mantidos por 24 horas e então removidos.

quando a inoculação ocorreu sobre folhas na planta (Tabela 5). Pode-se então concluir que o uso de folhas destacadas não é indicado para avaliação de resistência de soja a $S$. Sclerotiorum. As cultivares Emgopa 316 e BRSGO Milena, resistentes pelo método da folha destacada, comportaramse como moderadamente resistentes com a inoculação na planta. A cultivar FMT Tabarana, que foi a mais suscetível pelo método da folha destacada, comportou-se como moderadamente resistente quando a inoculação ocorreu na planta. Isso pode ser explicado pelo fato de que, quando a folha é destacada, os mecanismos de defesa da planta são interrompidos. Não houve correlação entre a severidade da doença nas cultivares Emgopa 316, BRS Milena, Emgopa 314, FMT Perdiz, FMT Tabarana, M-SOY 8360, BR 16, BRSMG 790A, P98R31, M-SOY 8352, M-SOY 2002, BRSMG 68 (Vencedora), BRSGO Princesa, CD 211, CD 205, BRSGO Caiapônia, M-SOY 8008, BRS 185, Emgopa 315 e BRS Baliza RR, quando comparadas às inoculações em folhas destacadas e em folhas na própria planta $(r=-0,11$, $\mathrm{p}>0,05)$. Possivelmente o número de cultivares utilizadas 
TABELA 4 - Reação de cultivares de soja a Sclerotinia sclerotiorum pelo método de inoculação em folha destacada

\begin{tabular}{|c|c|c|c|c|c|c|c|}
\hline Cultivares & $\begin{array}{l}\text { Cor da } \\
\text { flor }\end{array}$ & $\begin{array}{c}\text { Severidade } \\
(\%)\end{array}$ & Reação* & Cultivares & $\begin{array}{l}\text { Cor da } \\
\text { flor }\end{array}$ & $\begin{array}{c}\text { Severidade } \\
(\%)\end{array}$ & Reação \\
\hline FMT Tabarana & branca & $100,00 \mathrm{~h}$ & $\mathrm{~S}^{* *}$ & BRSMG 810C & branca & $91,67 \mathrm{~h}$ & $S$ \\
\hline TMG 115RR & branca & $100,00 \mathrm{~h}$ & $\mathrm{~S}$ & Emgopa 316 RR & branca & $91,00 \mathrm{~h}$ & S \\
\hline BR 4 & roxa & $100,00 \mathrm{~h}$ & $\mathrm{~S}$ & CD 219 & branca & $91,00 \mathrm{~h}$ & S \\
\hline FMT Tucunaré & branca & $100,00 \mathrm{~h}$ & $\mathrm{~S}$ & BRS Tracajá & roxa & $90,00 \mathrm{~h}$ & S \\
\hline BRS Sambaíba & branca & $99,33 \mathrm{~h}$ & $\mathrm{~S}$ & BRSGO Jataí & branca & $89,33 \mathrm{~h}$ & S \\
\hline BRSGO Chapadões & branca & $99,33 \mathrm{~h}$ & $\mathrm{~S}$ & BRSGO Raíssa & branca & $89,00 \mathrm{~h}$ & S \\
\hline BRS PampaRR & branca & $99,00 \mathrm{~h}$ & $\mathrm{~S}$ & Embrapa 48 & branca & $89,00 \mathrm{~h}$ & S \\
\hline Emgopa 31RR & branca & $98,67 \mathrm{~h}$ & $\mathrm{~S}$ & BRS 214 & branca & $88,67 \mathrm{~h}$ & S \\
\hline BRS 257 & branca & $98,00 \mathrm{~h}$ & $\mathrm{~S}$ & BRS Favorita & roxa & $88,00 \mathrm{~h}$ & S \\
\hline IAC 15 & branca & $98,00 \mathrm{~h}$ & $\mathrm{~S}$ & M-SOY 7908 & branca & $87,00 \mathrm{~h}$ & S \\
\hline BRSGO Araçu & branca & $98,00 \mathrm{~h}$ & $\mathrm{~S}$ & Nidera 7005 & roxa & $86,00 \mathrm{~h}$ & S \\
\hline BRSGO Santa Cruz & roxa & $97,33 \mathrm{~h}$ & $\mathrm{~S}$ & M-SOY 8045 & branca & $85,00 \mathrm{~h}$ & $\mathrm{~S}$ \\
\hline FT-Abyara & roxa & $97,00 \mathrm{~h}$ & $\mathrm{~S}$ & BRSGO Paraíso & roxa & $84,67 \mathrm{~g}$ & $\mathrm{~S}$ \\
\hline Cobb & branca & $97,00 \mathrm{~h}$ & $\mathrm{~S}$ & M-SOY 8384 RR & roxa & $84,00 \mathrm{~g}$ & S \\
\hline IAS 5 & branca & $97,00 \mathrm{~h}$ & $\mathrm{~S}$ & M-SOY 8045 RR & branca & $83,00 \mathrm{~g}$ & S \\
\hline BRS 212 & branca & $97,00 \mathrm{~h}$ & $\mathrm{~S}$ & M-SOY 8200 & branca & $82,00 \mathrm{~g}$ & S \\
\hline BRS 260 & branca & $97,00 \mathrm{~h}$ & $\mathrm{~S}$ & BRS Valiosa RR & roxa & $82,00 \mathrm{~g}$ & S \\
\hline TMG 113RR & branca & $97,00 \mathrm{~h}$ & $\mathrm{~S}$ & TMG 123 RR & branca & $81,67 \mathrm{~g}$ & S \\
\hline TMG 10 RR & roxa & $96,33 \mathrm{~h}$ & $\mathrm{~S}$ & BRS 133 & branca & $78,67 \mathrm{~g}$ & S \\
\hline BRSGO Mineiros & roxa & $96,33 \mathrm{~h}$ & $\mathrm{~S}$ & BRS Raimunda & branca & $57,67 \mathrm{f}$ & S \\
\hline CD 217 & roxa & $96,00 \mathrm{~h}$ & $\mathrm{~S}$ & M-SOY 8527 RR & roxa & $57,00 \mathrm{f}$ & $\mathrm{S}$ \\
\hline M-SOY 6101 & branca & $96,00 \mathrm{~h}$ & $\mathrm{~S}$ & BRSMG 850GRR & roxa & $49,67 \mathrm{e}$ & MS \\
\hline BRS 258 & branca & $96,00 \mathrm{~h}$ & $\mathrm{~S}$ & BRS Valiosa & roxa & $49,67 \mathrm{e}$ & MS \\
\hline Emgopa 302 & roxa & $96,00 \mathrm{~h}$ & $\mathrm{~S}$ & M-SOY 8000 RR & roxa & $49,00 \mathrm{e}$ & MS \\
\hline BRSGO Iara & branca & $95,67 \mathrm{~h}$ & $\mathrm{~S}$ & BRS Favorita RR & roxa & $28,67 \mathrm{~d}$ & MS \\
\hline BRS 213 & branca & $95,67 \mathrm{~h}$ & $\mathrm{~S}$ & BRSGO Luziânia & roxa & $28,00 \mathrm{~d}$ & MS \\
\hline BRS 164 & branca & $95,67 \mathrm{~h}$ & $\mathrm{~S}$ & Emgopa 314 (Garça Branca) & roxa & $19,00 \mathrm{c}$ & MS \\
\hline BRSGO-204 (Goiânia) & roxa & $95,33 \mathrm{~h}$ & $\mathrm{~S}$ & BRSMG 68 (Vencedora) & roxa & $19,00 \mathrm{c}$ & MS \\
\hline BRSGO MineirosRR & roxa & $95,33 \mathrm{~h}$ & $\mathrm{~S}$ & CD 205 & branca & $19,00 \mathrm{c}$ & MR \\
\hline BRS CharruaRR & branca & $95,33 \mathrm{~h}$ & $\mathrm{~S}$ & BRSGO Caiapônia & roxa & $18,33 \mathrm{c}$ & MR \\
\hline BRS 245RR & branca & $95,00 \mathrm{~h}$ & $\mathrm{~S}$ & CD 211 & branca & $17,33 \mathrm{c}$ & MR \\
\hline BRS 154 & branca & $95,00 \mathrm{~h}$ & $\mathrm{~S}$ & P98R31 & roxa & $16,33 \mathrm{c}$ & MR \\
\hline BRSGO Indiara & roxa & $94,67 \mathrm{~h}$ & $\mathrm{~S}$ & M-SOY 2002 & roxa & $15,00 \mathrm{c}$ & MR \\
\hline M-SOY 8199RR & roxa & $94,33 \mathrm{~h}$ & $\mathrm{~S}$ & BRSMG 790A & roxa & $13,67 \mathrm{~b}$ & MR \\
\hline BRSMG 752S & roxa & $94,33 \mathrm{~h}$ & $\mathrm{~S}$ & FMT Perdiz & roxa & $13,00 \mathrm{~b}$ & MR \\
\hline Nidera 7002 & roxa & $94,33 \mathrm{~h}$ & $\mathrm{~S}$ & Emgopa 315 & branca & $11,00 \mathrm{~b}$ & $\mathrm{R}$ \\
\hline BRSMG 750RR & branca & $94,00 \mathrm{~h}$ & $\mathrm{~S}$ & M-SOY 8360 & roxa & $10,67 \mathrm{~b}$ & $\mathrm{R}$ \\
\hline BRS 246 RR & branca & $93,67 \mathrm{~h}$ & $\mathrm{~S}$ & M-SOY 8352 & roxa & $10,33 \mathrm{~b}$ & $\mathrm{R}$ \\
\hline TMG 108RR & branca & $93,67 \mathrm{~h}$ & $\mathrm{~S}$ & BRS Baliza RR & branca & $10,00 \mathrm{~b}$ & $\mathrm{R}$ \\
\hline BRSMG Garantia & branca & $93,33 \mathrm{~h}$ & $\mathrm{~S}$ & M-SOY 8008 & branca & $9,33 \mathrm{~b}$ & $\mathrm{R}$ \\
\hline BRSMG 811CRR & roxa & $93,33 \mathrm{~h}$ & $\mathrm{~S}$ & BRS 185 & roxa & $8,33 \mathrm{~b}$ & $\mathrm{R}$ \\
\hline MG/BR-46 (Conquista) & roxa & $93,00 \mathrm{~h}$ & $\mathrm{~S}$ & BRSGO Princesa & roxa & $8,00 \mathrm{~b}$ & $\mathrm{R}$ \\
\hline BRS SilvâniaRR & branca & $92,33 \mathrm{~h}$ & $\mathrm{~S}$ & BRSGO Milena & roxa & $3,00 \mathrm{a}$ & $\mathrm{R}$ \\
\hline M-SOY 8001 & branca & $92,00 \mathrm{~h}$ & $\mathrm{~S}$ & BR 16 & branca & $1,33 \mathrm{a}$ & $\mathrm{R}$ \\
\hline BRSGO Ipameri & roxa & $91,67 \mathrm{~h}$ & $\mathrm{~S}$ & Emgopa 316 & roxa & $0,67 \mathrm{a}$ & $\mathrm{R}$ \\
\hline
\end{tabular}

$\mathrm{CV}(\%)$

7,40

Médias seguidas de letras distintas minúsculas nas colunas diferem entre si, pelo teste de Scott-Knott, a 1\% de probabilidade; *Resultados requerem confirmação em ensaios de campo, com incidência de doença acima de 35\%, segundo orientação da Comissão de Fitopatologia Reunião de Pesquisa de Soja do Brasil Central, Goiânia GO (18-20 de maio de 2009); **R = resistente, MR = moderadamente resistente, MS = moderadamente suscetível e $\mathrm{S}$ = suscetível;

na avaliação interfere no valor da correlação, em função da maior variabilidade das reações, quando comparado à situação envolvendo apenas duas cultivares contrastantes quanto à reação ao patógeno. A severidade entre as cultivares avaliadas variou de $20 \%$ (moderadamente resistente) a $95 \%$ (suscetível).

Nos estádios $V_{1}, V_{2}, V_{3}$ e $R_{1}$, os valores de severidade foram mais próximos entre as inoculações nos 
Avaliação da resistência da soja a Sclerotinia sclerotiorum em diferentes estádios...

TABELA 5 - Reação de cultivares de soja a Sclerotinia sclerotiorum pelo método de inoculação em folha na planta

\begin{tabular}{llcc}
\hline \hline Cultivares & Cor da flor & Severidade (\%) & Reação \\
\hline BRS Baliza RR & branca & $95,0 \mathrm{e}$ & $\mathrm{S}^{*}$ \\
Emgopa 315 & branca & $94,3 \mathrm{e}$ & $\mathrm{S}$ \\
BRS 185 & roxa & $90,0 \mathrm{e}$ & $\mathrm{S}$ \\
M-SOY 8008 & branca & $88,3 \mathrm{e}$ & $\mathrm{S}$ \\
BRSGO Caiapônia & roxa & $87,7 \mathrm{e}$ & $\mathrm{S}$ \\
CD 205 & branca & $87,3 \mathrm{e}$ & $\mathrm{S}$ \\
CD 211 & branca & $80,7 \mathrm{e}$ & $\mathrm{S}$ \\
BRSGO Princesa & roxa & $80,0 \mathrm{e}$ & $\mathrm{S}$ \\
BRSMG 68(Vencedora) & roxa & $60,0 \mathrm{~d}$ & $\mathrm{~S}$ \\
M-SOY 2002 & roxa & $55,0 \mathrm{~d}$ & $\mathrm{~S}$ \\
M-SOY 8352 & roxa & $48,3 \mathrm{c}$ & $\mathrm{MS}$ \\
P98R31 & roxa & $45,0 \mathrm{c}$ & $\mathrm{MS}$ \\
BRSMG 790 & roxa & $41,7 \mathrm{c}$ & $\mathrm{MS}$ \\
BR 16 & branca & $37,7 \mathrm{~b}$ & $\mathrm{MS}$ \\
M-SOY 8360 & roxa & $36,7 \mathrm{~b}$ & $\mathrm{MS}$ \\
FMT Tabarana & branca & $35,0 \mathrm{~b}$ & MS \\
FMT Perdiz & roxa & $32,7 \mathrm{~b}$ & MS \\
Emgopa 314 & roxa & $27,7 \mathrm{a}$ & MS \\
BRSGO Milena & roxa & $25,0 \mathrm{a}$ & MR \\
Emgopa 316 & roxa & $20,0 \mathrm{a}$ & MR \\
\hline CV (\%) & &
\end{tabular}

$\mathrm{CV}(\%)$

12,30

Médias seguidas de letras distintas minúsculas na coluna diferem entre si, pelo teste de Scott-Knott, a 1\% de probabilidade; $*$ S = suscetível, MS = moderadamente suscetível e MR = moderadamente resistente.

folíolos. Entretanto, considera-se que o estádio $\mathrm{V}_{1}$ seja ideal para inoculação, pois neste estádio os tecidos são tenros, o que facilita a infecção por S. sclerotiorum, patógeno de crescimento rápido. No estádio $R_{1}$, as plantas estão mais desenvolvidas e com tecidos mais lignificados, o que reduz a severidade de doença. Em campo, a planta se torna mais suscetível com o início do estádio $\mathrm{R}_{1}$, pois são nas flores que os ascósporos de $S$. sclerotiorum encontram fonte exógena de energia para germinar. Também nesta fase o microclima é mais favorável ao patógeno, devido ao maior índice de área foliar na fase pós-florescimento. Além disso, a maior cobertura foliar durante o fechamento da cultura permite que plantas doentes entrem em contato com plantas sadias, o que aumenta os focos da doença e/ou a sua disseminação radial. Com base nestes resultados, presume-se que a inoculação artificial reproduza com alguma segurança as infecções de campo (ocorrência natural) e evita possíveis escapes. A adoção da inoculação de S. sclerotiorum em plantas nos estádios $\mathrm{V}_{1}, \mathrm{~V}_{2}$ e $\mathrm{V}_{3}$ aceleraria os programas de seleção de genótipos de soja visando à resistência à podridão branca da haste, quando comparados à utilização de estádios mais maduros. Silva \& Machado (1989) consideraram o estádio $\mathrm{V}_{3}$ como apropriado para a inoculação de plantas de soja com $S$. sclerotiorum, durante o processo de seleção, enquanto que Chaves (1995) concluiu que o estádio $V_{1}$ é o mais indicado para a avaliação de variedades de soja quanto à resistência a $S$. sclerotiorum (utilizando a metodologia de micélio seco no colo da planta sem provocar ferimentos). Essas diferenças podem ser explicadas por causa das metodologias distintas empregadas por esses autores para inoculação, incluindo variações na forma de preparo e aplicação do inóculo.

Os métodos de "disco 24 horas" e "disco permanente" apresentaram resultados similares entre as cultivares e os órgãos inoculados (Tabelas 2 e 3). Estes resultados diferem dos obtidos por Cline \& Jacobsen (1983), que utilizaram a inoculação por tempo determinado, ou seja, contato rápido - conforme descrito por Hunter et al. (1981). Inoculação com disco de cultura foi um método prático e rápido, principalmente quando as inoculações ocorreram em folhas. Oliveira (1998), trabalhando com inoculação por disco de cultura na folha e palito contaminado na haste de plantas de feijão, verificou que o método de disco de BDA foi mais eficaz que o método de palito contaminado.

Os resultados obtidos com as cultivares FT Abyara, BR-4, BR-16 e IAS-5 (Tabela 4) foram diferentes dos obtidos por Chaves (1995), em relação à resistência a $S$. sclerotiorum, o que pode ser explicado pela diferença entre os isolados utilizados. No trabalho de Chaves (1995), FT Abyara foi mais resistente e Cobb mais suscetível em relação às demais cultivares. Isto pode ser explicado pelo fato de a inoculação ter sido feita com micélio seco e os isolados serem de localidades diferentes, denotando que pode ocorrer interação entre isolados de S. sclerotiorum e Glycine max. Variações relacionadas à interação entre isolados do patógeno e o hospedeiro também foram verificadas por Corradini (1989) com 19 isolados de S. sclerotiorum em Minas Gerais: os isolados causaram infecção na cultivar de 
soja Paraná, havendo variação na severidade da doença. Em estudos preliminares (dados não apresentados), o isolado oriundo de Jataí, GO, selecionado para este trabalho, foi mais agressivo que os isolados coletados em Indianópolis, MG e Presidente Olegário, MG. A resistência à doença parece não estar relacionada com a cor da flor, como sugerido por Grau et al. (1982), principalmente no que diz respeito à resistência de cultivares de flores roxas: de 41 cultivares, somente $13(32 \%)$ se comportaram como resistentes a moderadamente resistentes; cultivares de flores brancas também se comportaram como resistentes. As diferenças em relação ao trabalho de Zito et al. (2005) podem estar relacionadas à variabilidade genética na população do fungo, o que ainda não foi estudado em soja no Brasil. Este tipo de estudo é prioritário para o desenvolvimento de programas de melhoramento para resistência da soja ao patógeno. Devese considerar também que no trabalho de Zito et al. (2006) a incidência foi avaliada no campo, onde pode ocorrer escape à infecção em função do clima, arquitetura de plantas (Boland \& Hall, 1987), ciclo de maturação (Yang et al., 1999) e estádio fenológico. No presente trabalho evitou-se o escape com a inoculação artificial e a manutenção das plantas em câmara de crescimento a $22 \pm 3^{\circ} \mathrm{C}$, condições de extrema favorabilidade ao desenvolvimento da doença. No estudo realizado por Zito et al. (2005), o genótipo BR97-11548 e a cultivar Potenza foram mais suscetíveis à podridão branca da haste do que BRSMG Garantia, Monarca, MG/BR-46 (Conquista) e MGBR99-4656, no campo, em Sacramento, MG. Boland \& Hall (1987) verificaram diferença de suscetibilidade de 42 cultivares de soja no campo. Os autores observaram que a incidência da doença foi correlacionada com a altura da cultivar, severidade, maturidade e número de apotécios sob o dossel, indicando que o escape à doença é um mecanismo importante que afeta a incidência em algumas cultivares de soja. Yang et al. (1999) verificaram que a incidência de $S$. sclerotiorum em cultivares de soja está relacionada com os grupos de maturação. Segundo os autores, cultivares de ciclo longo são mais suscetíveis, devido ao maior período de florescimento, o que causa maior predisposição das plantas à infecção pelos ascósporos. A reação de 76 cultivares de soja em campo naturalmente infestado por S. sclerotiorum, foi avaliada por Homechin (1983). Nenhuma foi imune à doença, mas 13 apresentaram baixo número de plantas infectadas, denotando possível resistência. Silva \& Machado (1989) testaram 20 cultivares de soja à $S$. sclerotiorum através de inoculação artificial e verificaram que nenhuma cultivar foi imune ao final de 60 horas de incubação. Entretanto, as cultivares Numbaíra e IAC-11 apresentaram boa resistência, enquanto que UFV-1 e UFV-5 foram as mais suscetíveis ao patógeno.

Cline \& Jacobsen (1983) utilizaram a inoculação por tempo limitado desenvolvida por Hunter et al. (1981). O procedimento envolve a remoção precoce do inóculo (24 horas após a aplicação em sítio específico) para reduzir a severidade da doença e melhor revelar a resistência parcial que é superada com períodos mais longos de incubação.
Com esta técnica foi detectada diferença em resistência em 10 cultivares de soja. Quanto ao estádio de inoculação, Chun et al. (1987) estudaram os estádios $\mathrm{V}_{2}, \mathrm{~V}_{3}, \mathrm{~V}_{4}, \mathrm{~V}_{5}-\mathrm{V}_{6}$ e $V_{7}-R_{1}$ de plantas de soja inoculadas com $S$. sclerotiorum e verificaram que o desenvolvimento da doença decresceu com a idade da planta. Os autores sugeriram que fossem utilizadas plantas com até cinco semanas de idade para que diferenças entre cultivares fossem reveladas.

Foram encontradas, no presente trabalho, diferenças entre cultivares de soja quanto à suscetibilidade à podridão branca da haste. A utilização de cultivares de soja com resistência a $S$. sclerotiorum pode ser medida eficaz e econômica em locais onde a doença vem se destacando, como no Sudeste, Centro e Sul do Brasil. Juntamente com a resistência, medidas que promovam o escape à doença devem ser estudadas e incorporadas no manejo da doença. Novos estudos devem ser realizados em campo com inóculo natural e em condições de alta severidade da doença para confirmar os resultados obtidos. Da mesma forma, estudos são necessários com inoculação artificial de ascósporos durante o florescimento das plantas.

\section{AGRADECIMENTOS}

Este trabalho é parte da Dissertação de Mestrado de R. A. Garcia (Universidade Federal de Uberlândia, MG, 2008), com bolsa de estudo financiada pelo Conselho Nacional de Desenvolvimento Científico e Tecnológico CNPq.

\section{REFERÊNCIAS BIBLIOGRÁFICAS}

Almeida AMR, Pereira LP, Yorinori JT, Silva JFV, Henning CV, Godoy LM, Costamilan LM, Meyer MC (2005) Doenças da Soja. In: Kimati H, Amorim L, Rezende JAM, Bergamin Filho A, Camargo LEA (Eds.) Manual de Fitopatologia. Vol. 2. Doenças das Plantas Cultivadas. $4^{\text {a }}$. Ed. São Paulo SP. Ceres. pp. 569-588.

Boland GJ, Hall R (1987) Evaluating soybean cultivars for resistance to Sclerotinia sclerotiorum under field conditions. Plant Disease 71:934-936.

Chaves MS (1995) Aspectos epidemiológicos da interação Whetzelinia sclerotiorum x Glycine max. Dissertação de Mestrado. Universidade Federal do Rio Grande do Sul. Porto Alegre RS.

Chaves MS, Martinelli JA, Loch LC (1996) Uso de micélio seco de Sclerotinia sclerotiorum como método de inoculação e avaliação da resistência de cultivares de soja. Summa Phytopathologica 22:221-224.

Chun D, Kao LB, Lockwood, JL (1987) Laboratory and field assessment of resistance in soybean to stem rot caused by Sclerotinia sclerotiorum. Plant Disease 71:811-815.

Cline MN, Jacobsen, BJ (1983) Methods for evaluating soybean cultivars for resistance to Sclerotinia sclerotiorum. Plant Disease 67:784-786.

Corradini HT (1989) Características em cultura pura e patogenicidade de isolados de Sclerotinia sclerotiorum (Lib.) de Bary associados à soja (Glycine Max (L.) Merril na região do 
Alto Paranaíba, MG. Dissertação de Mestrado. Escola Superior de Agricultura de Lavras. Lavras MG.

Garcia RA, Juliatti, FC (2008) Reação de genótipos de soja ao mofo branco causado por Sclerotinia sclerotiorum. Tropical Plant Pathology 33:213. (Resumo)

Grau CR, Radke VL, Gillespie, FL (1982) Resistance of soybean cultivars to Sclerotinia scleroitorum. Plant Disease 66:506-508.

Homechin M (1983) Reação de cultivares comerciais de soja a Sclerotinia sclerotiorum. Fitopatologia Brasileira 8:559. (Resumo)

Hunter JE, Dickson, MH, Cigna JA (1981) Limited-term inoculation: A method to screen bean plants for partial resistance to white mold. Plant Disease 65:414-417.

Juliatti FC, Juliatti FCA (2010) Podridão branca da haste da soja: Manejo e uso de fungicidas em busca da sustentabilidade nos sistemas de produção. Uberlândia MG. Composer.

Leite RMVBC (2005) Ocorrência de doenças causadas por Sclerotinia sclerotiorum em girassol e soja. Embrapa Soja. Londrina PR. (Comunicado Técnico 76)

Oliveira SHF (1998) Controle químico de Sclerotinia sclerotiorum em feijoeiro: Ação in vitro sobre o ciclo de vida, ação preventiva e curativa em condições controladas e eficiência e modo de aplicação em campo. Tese de Doutorado. Escola Superior de Agricultura "Luiz de Queiroz". Piracicaba SP.

Silva SM, Machado JC (1989) Metodologia de inoculação e comportamento de alguns cultivares de soja (Glycine max L.), em relação a Sclerotinia sclerotiorum. Fitopatologia Brasileira 14:118. (Resumo)

Vale FXR, Fernandes Filho ER, Liberato JR (2003) QUANT - A software for plant disease severity assessment. In: Proceedings of the $8^{\circ}$ International Congress of Plant Pathology. Christchurch, New Zealand. p. 105.

Wegulo SN, Yang XB, Martinson CA (1998) Soybean cultivar responses to Sclerotinia sclerotiorum in field and controlled environment studies. Plant Disease 82:1264-1271.

Yang XB, Lundeen P, Uphoff D (1999). Soybean varietal response and yield loss caused by Sclerotinia sclerotiorum. Plant Disease $83: 456-461$.

Zito RK, Wruck DSM, Fronza V, Arantes NE (2005) Reação de genótipos de soja a Sclerotinia sclerotiorum. In: Anais da XXVII Reunião de Pesquisa de Soja da Região Central do Brasil. Cornélio Procópio PR. p. 362.

TPP 107 - Recebido 14 Abril 2010 - Aceito 13 Abril 2012 Editor de Seção: Eduardo S. Mizubuti 